\title{
Understanding Bug Fix Patterns in Verilog
}

\author{
Sangeetha Sudhakrishnan \\ sangeetha@soe.ucsc.edu
}

\author{
Janaki T. Madhavan \\ janaki@soe.ucsc.edu \\ Jose Renau \\ renau@soe.ucsc.edu
}

\author{
E. James Whitehead Jr. \\ ejw@soe.ucsc.edu
}

\author{
University of California, Santa Cruz CA
}

\begin{abstract}
Today, many electronic systems are developed using a hardware description language, a kind of software that can be converted into integrated circuits or programmable logic devices. Like traditional software projects, hardware projects have bugs, and significant developer time is spent fixing them. A useful first step toward reducing bugs in hardware is developing an understanding of the frequency of different types of errors. Once the most common types are known, it is then possible to focus attention on eliminating them. As most hardware projects use software configuration management repositories, these can be mined for the textual bug fix changes. In this project, we analyze the bug fix history of four hardware projects written in Verilog and manually define 25 bug fix patterns. The frequency of each bug type is then computed for all projects. We find that $29-55 \%$ of the bug fix pattern instances in Verilog involve assignment statements, while $18-25 \%$ are related to if statements.
\end{abstract}

Categories and Subject Descriptors: D.2.4 [Software]: Program Verification

General Terms: Languages, Design

Keywords: Fault, bug, fix, Patterns.

\section{INTRODUCTION}

During the 1990's, the design of integrated circuits shifted from the traditional labor intensive process of schematic layout towards the use of hardware description languages (HDLs), such as Verilog and VHDL. Similar to the tradeoff of early programming languages, HDLs produced less efficient ciruits, but at higher designer productivity. One consequence is that hardware development now has a heavy software development flavor.

Verilog is one of the most widely used hardware description languages. The language supports the design, verification, and implementation of analog, digital, and mixed-signal circuits at various levels of abstraction. It is similar to the $\mathrm{C}$ programming language since its preprocessor and control

Permission to make digital or hard copies of all or part of this work for personal or classroom use is granted without fee provided that copies are not made or distributed for profit or commercial advantage and that copies bear this notice and the full citation on the first page. To copy otherwise, to republish, to post on servers or to redistribute to lists, requires prior specific permission and/or a fee.

MSR'08, May 10-11, 2008, Leipzig, Germany.

Copyright 2008 ACM 978-1-60558-024-1/08/05 ...\$5.00. flow keywords are C-like. The language differs from $\mathrm{C}$ in the following. It uses Begin/End to specify a block instead of the curly braces. The definition of constants requires a bit width along with their base and Verilog does not have structures, pointers, or recursive subroutines. The concept of time is important in HDLs, and is not found in C. Another major difference from a conventional language is that the statements are not executed strictly linearly.

Just as in software, a hardware project goes through design, development, and testing phases. A designated team of testers usually checks whether the project exhibits the required external behavior. When undesirable behavior is detected, a log is maintained, similar to bug tracking in software projects. This undesirable behavior is called a bug and fixing the bug involves modification of the HDL source code. Software configuration management repositories are widely used to record the evolution of hardware projects. Similar to SourceForge, there is a publically available repository of open source HDL software, www.opencores.org, that contains over 450 HDL projects including arithmetic cores, communication controllers, memory cores, video controllers, and microprocessor cores. As in software projects, changes made to HDL projects include a brief description of the change, recorded in the SCM change log. More often than not, words like "fixed" and "bug" are used to describe the changes related to bug fixes. Hence, these repositories can be mined for log messages containing these words to obtain the bug fix changes. Indeed, it appears that many of the techniques developed to exploit information found in the development history of software projects can be applied to the development history of HDL projects too.

Given this similarity between HDL and high-level programming languages, one question that arises is whether the types and frequencies of bugs in an HDL like Verilog are similar to those in, say, Java. In prior work, Kai Pan manually developed a series of fine-grained bug fix patterns in Java software [1]. These bug fix patterns are automatically extractable from the history of a software project. The bug fix patterns can be viewed as a kind of error taxonomy. Unlike existing work to categorize software errors into specific categories, the bug fix patterns are automatically categorized; prior work involved a human manually performing the categorization work, resulting in poor scalability and unreliable categorizations. This paper reports on an effort to translate this idea of bug fix patterns over to Verilog.

We present a series of bug fix patterns observed in four open source Verilog projects The patterns we found were seen to be related to assignments (blocking, non-blocking and as- 
sign), "if" statements, module declaration and instantiation, "always" and "switch" statements. We have obtained the frequency of their occurence in the four projects we have analyzed. Errors in assignment were found to be the most common pattern, followed by the "if" related ones. In order to understand them in greater detail, we have characterized them further into many sub-patterns.

The remainder of the paper is organized as follows. Section II discusses the methodology used to obtain the bug fix changes from the change history of the projects analyzed. Section III describes the identified Verilog bug fix patterns in detail. The frequency of the observed patterns in the four projects are presented in Section IV. Section VI discusses the related work in this area. And finally, Section VII presents ideas for future work and concludes the report.

\section{METHODOLOGY FOR IDENTIFYING BUG FIXES}

In this paper, we use the term bug for a mistake made in the source code by the developer, which results in an undesirable external behaviour during the execution of the code. When the developers commit the changes that repair a bug to the software configuration management repository, they usually log a brief note about these changes. The bug fixes are typically denoted by the words "bug" and "fixed". Therefore, by going over the change history, we can obtain the revision that contains the changes that fix a bug (bug fix revision) and the one that has the bug (buggy).

There are two main approaches to get the bug fix revisions and their corresponding buggy revisions. One of the techinques involves looking for references to bug reports; this was introduced by Mockus and Votta [2]. The other one, introduced by Fischer et al [3] and by Cubranic and Murphy [4], involves scanning the change logs for keywords like "fixed" or "bug"; the revisions, whose change logs contain these words are considered to be the bug fix revisions and the revisions prior to them are their respective buggy revisions. We follow the second approach and manually look for the above mentioned keywords in the change logs of the Verilog projects, we analyzed. After obtaining the buggy and the bug fix revisions, we compute the changes to each file involved in the latter by doing a "diff" with their counterparts in the former. Adopting the terminology used by [1], we call this textual difference a bug fix hunk pair. A bug hunk refers to the code section in the buggy version, that is modified or deleted in the bug fix revision. The corresponding fix hunk comprises the modifications in the bug fix version that fixes the bug. These two together form the bug fix hunk pair. A bug fix change is typically a modification, addition or deletion of some specific lines of the source code.

To get some insight into the kind of changes developers make to repairs bugs in Verilog, we have looked for syntax-based patterns in the bug fix hunk pairs. The patterns found by us are explained in detail in the next section.

\section{BUG FIX PATTERNS}

To obtain all the bug fix patterns in Verilog, we manually analyzed the four open source projects downloaded from www.opencores.org. They are listed in Table I. By following the methodology described in the previous section, we obtained the bug fix hunk pairs and identified the bug fix patterns present in them. These patterns are grouped
Table 1: Projects Analyzed

\begin{tabular}{|c|c|c|}
\hline Project Name & \# of Revisions & \# of Bugs \\
\hline \hline Uart_16550 & 32 & 128 \\
OC_8051 & 22 & 121 \\
OR1K & 17 & 108 \\
Mem_ctrl & 45 & 169 \\
\hline
\end{tabular}

into the following major categories: if-related (IF), moduledeclaration (MD), module-instantiation (MI), assignment(A $\mathrm{S})$, switch (SW), always (AL) and class field (CF). Examples of the bug patterns are shown below. Due to lack of space we explain only 2 patterns. A more detailed explanation can be found at [14]. Using code snippets from these projects, two of the 25 patterns is explained in detail below. In the code snippets provided as examples, the lines that begin with - belong to the buggy revision, while the lines that begin with + belong to the bug fix revision.

1. Addition of an if statement (IF_APC): This pattern is observed when an if statement is added to fix a bug. This statement is usually added to ensure that a certain condition is met before performing an operation.

Example:

$$
+\quad \text { if }(r s t) m a \_i s s u e \leq 1^{\prime} b 0
$$

2. Addition of "assign" statement (ASG_ADD): An assign statement is used to model combinatonal logic, and this pattern occurs when additional logic previously not included in the code is added to the existing logic Example:

$$
+\quad \text { assign mux_out }=\text { sel? } a: b \text {; }
$$

We have ignored changes related to comments, synthesis statements, include statements, param statements, code cleanup and code formatting. Excluding these, we have classified all the changes observed in the four projects and the results are summarized in Table 2 . In the next section, we characterize the bug fix patterns obtained in these projects.

\section{CHARACTERISTICS OF BUG FIX PATTERNS}

In this section, we show the frequency of bug fix patterns in the four verilog projects analyzed. Figure 1 gives the frequencies of all the bug fix patterns across projects.

It is clear that the assignment related patterns are the most common. They account for $29-55 \%$ of all observed patterns. This is followed by the if related patterns. They account for $18-25 \%$ of the observed patterns. The combined frequency of module declaration and module instantiation is seen to be $4.7-25 \%$. The class field related patterns occur with frequency $4.7-16 \%$. The frequency of the always related patterns is about $2.8-21 \%$. And finally, case related patterns account for only about 1.8-4.6\%. Apart from the assignment and the if related patterns, which follow similar trend across all the four projects (most common and second most common respectively), we cannot say anything conclusive about the rest of the projects. 


\begin{tabular}{|c|c|c|c|c|c|c|c|c|c|}
\hline CATEGORY & PATTERN NAME & SHORTNAME & UART 16550 & \multicolumn{2}{|c|}{$0 \mathrm{C} 8051$} & \multicolumn{2}{|r|}{ OR1K } & \multicolumn{2}{|c|}{ MEM_CTRL } \\
\hline & & & $\%$ & \# & $\%$ & \# & $\%$ & \# & $\%$ \\
\hline \multirow[t]{6}{*}{ IF } & Addition of else related branch & IF_ABR & 1.56 & 12 & 10 & $\overline{9}$ & 8.3 & 6 & 3.5 \\
\hline & Addition of if branch & IF_APC & 4.7 & $\overline{2}$ & 1.7 & $\overline{0}$ & 0 & 12 & 7.1 \\
\hline & Change of if condition expression & IF_CC & 11 & $\overline{9}$ & 7.4 & 6 & 5.5 & 20 & 11.8 \\
\hline & Removal of else branch & IF_RBR & 3.1 & 1 & 0.8 & 3 & 2.8 & 1 & 0.6 \\
\hline & Removal of if branch & IF_RMV & 4.6 & $\overline{3}$ & 2.5 & 1 & 0.92 & 4 & 2.3 \\
\hline & TOTAL & & 24 & 27 & 22.3 & 19 & 18 & 43 & 25 \\
\hline \multirow[t]{3}{*}{ MI } & Module instantiation with different number of ports & MI_DNP & 1.56 & $\overline{4}$ & 3.3 & 7 & 6.5 & 3 & 1.8 \\
\hline & Module instantiation with different values for the ports & MI_DCP & 0 & 2 & 1.65 & $\overline{9}$ & 8.3 & 7 & 4.1 \\
\hline & $\begin{array}{ll}-20 T A L \\
\end{array}$ & & 1.6 & 6 & 4.9 & 16 & 15 & 10 & 6 \\
\hline \multirow[t]{2}{*}{ MD } & Module declaration with different number of ports & MD_DNP & 3.1 & 5 & 4.1 & 11 & 10 & 7 & 4.1 \\
\hline & TOTAL & & 3.1 & 5 & 4.1 & 11 & 10 & 7 & 4.1 \\
\hline \multirow[t]{11}{*}{ AS } & Change of expression for non_blocking assignment & NB_CE & 16.4 & 9 & 7.4 & 6 & 5.5 & 5 & 3 \\
\hline & Change of expression for blocking assignment & B_CE & 0 & 4 & 3.3 & 1 & 0.92 & 6 & 3.5 \\
\hline & Change of expression for assign statement & ASG_CE & 4.7 & 13 & 10.7 & 27 & 25 & 5 & 2.95 \\
\hline & Change of expression for assign statement & ASG_CE & 4.7 & 13 & 10.7 & 27 & 25 & 5 & 2.95 \\
\hline & Assign statement added & ASG_ADD & 21.8 & 5 & 4.1 & $\overline{4}$ & 3.7 & 7 & 4.1 \\
\hline & Assign statement removed & ASG_RMV & 3.1 & 5 & 4.1 & 1 & 0.92 & 8 & 4.73 \\
\hline & Non_blocking statement added & NB_ADD & 7.8 & 5 & 4.1 & 1 & 0.92 & 3 & 1.78 \\
\hline & Non_blocking statement removed & NB_RMV & 0.8 & 8 & 6.6 & 0 & 0 & 0 & 1.18 \\
\hline & Blocking statement added & B_ADD & 0 & 3 & 2.5 & 0 & 0 & 7 & 4.14 \\
\hline & Blocking statement removed & B_RMV & 0 & 1 & 0.8 & $\overline{0}$ & 0 & 6 & 3.55 \\
\hline & TOTAL & & 54.6 & 53 & 44 & 40 & 37 & 49 & 29 \\
\hline \multirow[t]{2}{*}{ SW } & Addition or removal of case branches & SW_ABRP & 4.6 & 3 & 2.5 & $\overline{2}$ & 1.9 & 3 & 1.77 \\
\hline & TOTAL & & 4.6 & 3 & 2.5 & 2 & 1.9 & 3 & 1.77 \\
\hline \multirow[t]{5}{*}{$\mathbf{C F}$} & Addition of reg & RG_ADD & 3.1 & 10 & 8.26 & 5 & 4.7 & 11 & 6.5 \\
\hline & Addition of wire & WR_ADD & 0.8 & 4 & 3.3 & 8 & 7.4 & 7 & 4.14 \\
\hline & Removal of reg & RG_RMV & 0.8 & 4 & 3.3 & 1 & 0.92 & 0 & 0 \\
\hline & Removal of wire & WR_RMV & 0 & 0 & 0 & 3 & 2.8 & 4 & 2.366 \\
\hline & TOTAL & & 4.7 & 18 & 15 & 17 & 16 & 22 & 13 \\
\hline \multirow[t]{5}{*}{ AL } & Sensitivity list changes & $\mathrm{AL} \_\mathrm{SE}$ & 0.8 & 2 & 1.65 & 1 & 0.92 & 11 & 6.5 \\
\hline & Always added & AL_ADD & 4.6 & 5 & 4.1 & 2 & 1.9 & 20 & 11.83 \\
\hline & Always removed & AL_RMV & 3.1 & 2 & 1.65 & & 0 & 4 & 2.36 \\
\hline & TOTAL & & 7.8 & 9 & 7.5 & 3 & 2.8 & 35 & 21 \\
\hline & TOTAL & & 128 & 121 & & 108 & & 169 & \\
\hline
\end{tabular}

Table 2: The count and frequency of the extracted pattern instances across projects

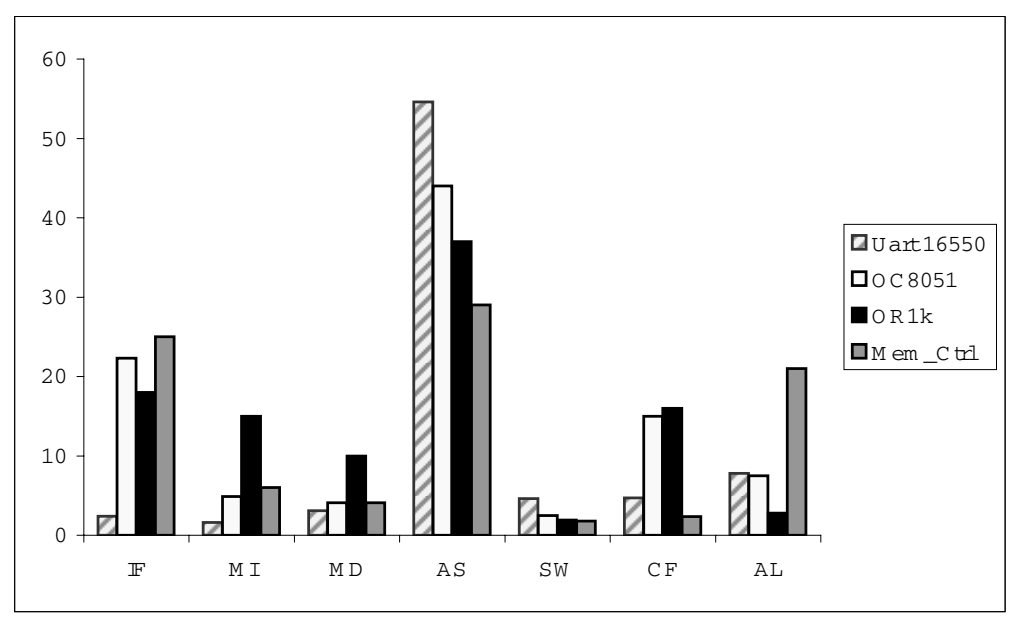

Figure 1: Bar chart of the frequency distribution of bugs in the projects analyzed 


\section{RELATED WORK}

Bugs in software are difficult to find and costly to fix. Therefore, static code analysis to detect bugs is a very active area of research in software engineering.

The closest work to the work presented in this paper is by [9]. The authors study 4 student hardware design projects and present error distributions. The major difference with the work presented here is that designers were asked to create new revision via CVS whenever a design error was corrected or whenever the design process was interrupted. In this paper, we mine the revision history after the project was created and looked for revision logs containing the words "Bug" or "Fix", thereby considering only changes made to code to correct a design bug. Further, the taxonomy used by Campenhout et al. [9] is ambiguous when compared to the taxonomy presented in this paper.

Many techniques have been developed over the years to automatically detect bugs in software. These techniques are usually based on syntactic pattern matching, data flow analysis, type systems and theorem proving. FindBugs [5], JLint [6, 7], ESC/Java 2 [8], PMD [10] are some examples. These tools are applied to find bugs in Java, which has many potential coding pitfalls [11]. There has also been work that compares the effectiveness of these tools [13]. All these tools are based on static analysis and the bug patterns are based on known program errors. These tools do not use the change history of a software project to find the real bugs reported by the developers.

FindBugs is a tool to detect bug patterns in Java. Its main technique is to analyze the bytecode and syntactically match source code to known suspicious programming practice. This approach is similar to ASTLog [12]. It also uses data flow analysis to look for bugs. JLint is also based on analysing bytecode, performing syntactic checks and dataflow analysis. PMD also performs syntactic checks on program source code and does not have the dataflow component. ESC/Java is based on theorem proving and performing formal verifications of properties of Java source code.

The change history of a software project can give considerable information about the common bugs and the patterns found in their fixes. This information has been exploited to develop an extractor tool to automatically extract 27 bug fix patterns in Java projects [1]. All the tools described above have been developed for Java; to our knowledge, there has been no work on statically analyzing bugg code patterns in hardware description languages. Our approach, based on [1], looks for the bug fix patterns in the software change history of Verilog projects. We have thus categorized all the observed bug-fix related changes into 25 bug fix patterns.

\section{CONCLUSIONS AND FUTURE WORK}

In this paper, we obtained the bug fix patterns in a hardware description language, Verilog, observing 25 categories of bug fix patterns. Among them, the assignment related patterns are found to be the most common, followed by if related ones. The former accounts for $29-55 \%$ of all observed patterns, while the latter accounts for $18-25 \%$. In contrast, the bug fix patterns in Java are dominated by the if statements and the method calls [1]. They account for 45-60.3\% of all defined bug fix patterns. The correlation analysis on the extracted pattern instances on the four projects indicates that most of them have similar bug fix pattern distributions.
We could only examine four Verilog projects as our analysis was manual. Also, finding Verilog projects with long history of evolution was difficult. In our future work, we would like to examine more projects to investigate whether the trend found in this paper holds across other projects. If it does, then it should be possible to leverage this pattern knowledge in the creation of static analysis bug finders for Verilog. These patterns can be used to develop bug prediction mechanisms for Verilog. A similar analysis can be done for other hardware description languages to see whether the bug fix patterns are similar to those of Verilog.

\section{REFERENCES}

[1] Pan, Kai, "Using Evolution Patterns to Find Duplicated Bugs", Ph.D Dissertation, Computer Science, UC Santa Cruz, 2006. Available at http://www.soe.ucsc.edu/ ejw/ dissertations/pan-dissertation.pdf

[2] A. Mockus and L. G. Votta, Identifying Reasons for Software Changes Using Historic Database, In Proceedings of International Conference on Software Maintenance (ICSM 2000), San Jose, California, 2000, pp 120-130.

[3] M.Fischer, M. Pinzger and H. Gall, Populating a Release History Database from Version Control and Bug Tracking Systems, In Proceedings of 2003 International Conference on Software Maintenance (ICSM'03), Amsterdam, Netherlands, 2003, pp 23-32.

[4] D. Cubranic and G. C. Murphy, Hipikat: Recommending Pertinent Software Development Artifacts, In Proceedings of the 25th International Conference on Software Engineering, Portland, Oregon, 2003, pp 408-418.

[5] D. Hovemeyer and W. Pugh, Finding Bugs is Easy, ACM SIGPLAN Notices, vol. 39, no. 12, pp 92-106, 2004.

[6] C. Artho, Finding Faults in Multi-threaded Programs, Master's Thesis, Institute of Computer Systems, Federal Institute of Technology, Zurich/Austin, 2001.

[7] JLint, http://artho.com/jlint .

[8] C. Flanagan, K. R. M. Leino, M. Lillibridge, G. Nelson, J. B. Saxe, R. Stata, Extended Static Checking for Java, In Proceedings of the 2002 ACM SIGPLAN Conference on Programming Language Design and Implementation, pp 234-245, Berlin, Germany, June 2002.

[9] D. Van Campenhout and H. Al-Asaad and J. Hayes and T. Mudge and R. Brown, High-level design verification of microprocessors via error modeling, Trans. Design Automation of Electronic Systems, 3(4):581-599, October 1998

[10] PMD/Java, http://pmd.sourceforge.net

[11] E. Allen, Bug Patterns in Java, APress, 2002.

[12] R. F. Crew, ASTLOG: A Language for Examining Abstract Syntax Trees, In Proceedings of the Conference on Domain-Specific Languages, Santa Barbara, California, Oct 1997.

[13] N. Rutar, C. B. Almazan and J. S. Foster, A Comparison of Bug Finding Tools for Java, in The 15th IEEE International Symposium on Software Reliability Engineering (ISSRE'04), pp 245-256, Saint-Malo, Bretagne, France, November 2004.

[14] S.Sudhakrishnan, J.Madhavan, E.J.Whitehead, J.Renau. Understanding Bug Fix Patterns in Verilog Available at

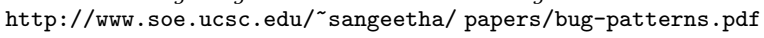

